PINHEIRO JB; REIFSCHNEIDER FJB; PEREIRA RB; MOITA AW. 2014. Reação de genótipos de Capsicum ao nematoide-das-galhas. Horticultura Brasileira 32: 371-375. DOI - http://dx.doi.org/10.1590/S0102-05362014000300022

\title{
Reação de genótipos de Capsicum ao nematoide-das-galhas
}

\section{Jadir B Pinheiro; Francisco JB Reifschneider; Ricardo B Pereira; Antonio W Moita}

Embrapa Hortaliças, C. Postal 218, 70351-000 Brasilia-DF; jadir.pinheiro@embrapa.br; francisco.reifschneider@embrapa.br; ricardoborges.pereira@embrapa.br; antonio.moita@embrapa.br

\section{RESUMO}

O objetivo desta pesquisa foi avaliar a reação de híbridos porta-enxerto experimentais de Capsicum annuum ['Hibrido Experimental (HE)' e seu Recíproco (HER)] e genótipos elite do programa de melhoramento da Embrapa Hortaliças de $C$. chinense, $C$. frutescens e C. baccatum à Meloidogyne incognita raça $1, M$. javanica e $M$. enterolobii. Como testemunha suscetível a $M$. incognita raça 1 e resistente a $M$. javanica foi utilizado o híbrido de pimentão 'Magali $\mathrm{R}$ ', e como testemunhas resistentes a $M$. incognita raça 1 e $M$. javanica foram utilizados os porta-enxertos 'Silver' e 'Snooker'. Para confirmar a viabilidade do inóculo foi utilizado o tomateiro 'Rutgers'. Dois experimentos foram conduzidos em casa de vegetação, em delineamento inteiramente casualizado com seis repetições. Plântulas com 30 dias de idade foram transplantadas para vasos de 2,0 L contendo substrato composto de solo, areia, esterco de gado e palha de arroz carbonizada $(1: 1: 1: 1)$. As plantas foram inoculadas após o transplantio com a deposição de $5,0 \mathrm{~mL}$ de suspensão, contendo 5.000 ovos e J2 de cada espécie. Setenta dias após foram avaliados o índice de massa de ovos e de galhas, o número de ovos por grama de raiz e o fator de reprodução. Os híbridos de C. annuum apresentaram resistência a $M$. incognita e $M$. javanica e suscetibilidade a M. enterolobii, assim como as testemunhas 'Silver' e 'Snooker'. O híbrido 'Magali R' apresentou resistência somente a $M$. javanica. Os genótipos de $C$. chinense e $C$. baccatum apresentaram suscetibilidade às três espécies, enquanto genótipos de $C$. frutescens apresentaram suscetibilidade somente a $M$. enterolobii. Os híbridos experimentais de pimentão e a maioria dos genótipos de $C$. frutescens avaliados poderão ser utilizados em áreas de cultivo protegido infestadas com $M$. incognita raça 1 e M. javanica. Entretanto, a busca por genótipos resistentes a $M$. enterolobii, bem como o desenvolvimento e a adoção de estratégias de controle é de suma importância.

Palavras-chave: Meloidogyne incognita, Meloidogyne javanica, Meloidogyne enterolobii, resistência, fator de reprodução, pimenta.

\section{ABSTRACT \\ Reaction of Capsicum genotypes to root-knot nematode}

The objective of this research was to evaluate the response of experimental hybrids of Capsicum annuum ['Experimental Hybrid (EH)' and its Reciprocal (EHR)] and Embrapa Hortaliças elite genotypes of $C$. chinense, $C$. frutescens and $C$. baccatum to Meloidogyne incognita race 1, M. javanica and M. enterolobii. The hybrid pepper 'Magali R' was used as susceptible control to $M$. incognita race 1 and resistant to $M$. javanica, and as resistant controls to M. incognita race 1 and M.javanica 'Silver' and 'Snooker' rootstocks were used. 'Rutgers' tomato was used to confirm the viability of the inoculum. Two experiments were carried out in a greenhouse in a completely randomized design with six replications. Thirty-day old seedlings were transplanted to $2.0 \mathrm{~L}$ pots containing a mixture of soil, sand, cattle manure and carbonized rice husk (1:1:1:1). Plants were inoculated after transplanting with deposition of $5.0 \mathrm{~mL}$ of suspension containing 5,000 eggs and $\mathrm{J} 2$ of each species alone. After seventy days, plants were assessed by measuring the index of egg mass and galls, the number of eggs per gram of roots and the reproduction factor. The $C$. annuum experimental rootstock hybrids were resistant to $M$. incognita and $M$. javanica and susceptible to M. enterolobii, similar to 'Silver' and 'Snooker'. The hybrid 'Magali $\mathrm{R}$ ' only showed resistance to $M$. javanica. Elite genotypes of $C$. chinense and $C$. baccatum were susceptible to all three species, while genotypes of $C$. frutescens were susceptible only to M. enterolobii. The experimental hybrids of Capsicum annuum and most genotypes of $C$. frutescens may be used in protected cultivation areas infested with $M$. incognita race 1 and M. javanica. However the search for resistant genotypes to $M$. enterolobii, as well as the development and adoption of control strategies is of crucial importance.

Keywords: Meloidogyne incognita, Meloidogyne javanica, Meloidogyne enterolobii, resistance, reproduction factor, pepper.

\section{(Recebido para publicação em 3 de julho de 2013; aceito em 20 de junho de 2014) (Received on July 3, 2013; accepted on June 20, 2014)}

$\mathrm{O}$ cultivo do pimentão (Capsicum annuum) em estufa tem aumentado consideravelmente nos últimos anos devido às altas produtividades $\mathrm{e}$ rentabilidade da cultura. Entretanto, o cultivo intensivo neste sistema tem acarretado graves problemas, destacando-se a maior ocorrência de doenças devido à limitação de rotação de culturas. Nestas condições, patógenos habitantes de solo são favorecidos e se estabelecem na área após cultivos sucessivos de materiais suscetíveis, como é o caso dos nematoides-das-galhas, especialmente Meloidogyne incognita e M. javanica, que são mais parasitos das hortaliças.
Outra espécie de nematoide-das-galhas, Meloidogyne enterolobii (sin. Meloidogyne mayaguensis), tem causado prejuízos no cultivo de algumas hortaliças. Esta espécie foi detectada pela primeira vez no Brasil em Petrolina-PE e Curaçá-BA e Maniçoba-BA, causando severos danos em plantios de goiabeira 
(Carneiro et al., 2001). Em hortaliças, esta espécie foi relatada pela primeira vez no estado de São Paulo parasitando o porta-enxerto de pimentão 'Silver' e tomateiros 'Andréa' e 'Débora', resistentes à $M$. incognita e $M$. javanica (Carneiro et al., 2006).

Atualmente encontram-se disponíveis comercialmente híbridos intraespecíficos de pimentão porta-enxerto denominados 'Silver' (Sakata, 2012) e 'Snooker' (Syngenta, 2012), ambos com resistências a $M$. incognita raças $1,2,3$ e 4 e $M$. javanica de acordo com as informações das duas empresas. Contudo, estes apresentam suscetibilidade a M. enterolobii.

A utilização de porta-enxertos resistente a nematoides do gênero Meloidogyne e a outros patógenos de solo tem sido recomendada por apresentar bons resultados no controle de doenças em cultivos protegidos, principalmente de tomate, pepino, melão, berinjela e pimentão (Santos, 2003). Por ser uma técnica de fácil adoção em função da pequena interferência no manejo já utilizado pelos produtores, o uso de porta-enxertos apresenta-se como eficiente estratégia de controle de doenças.

Este trabalho teve como objetivo avaliar a reação de híbridos experimentais de C. annuum em desenvolvimento na Embrapa Hortaliças e genótipos elite de pimenta $C$. chinense, $C$. frutescens e $C$. baccatum, também desenvolvidos pelo Programa de Melhoramento de Capsicum da Embrapa, a $M$. incognita raça $1, M$. javanica e $M$. enterolobii.

\section{MATERIAL E MÉTODOS}

O presente trabalho foi realizado em casa de vegetação e no Laboratório de Nematologia da Embrapa Hortaliças, Brasília-DF, no período de maio a agosto de 2009.

A identificação das espécies de nematoides-das-galhas, $M$. javanica, $M$. incognita raça 1 e $M$. enterolobii, foi realizada mediante corte da região perineal de fêmeas adultas, com base nos padrões descritos por Yang \& Eisenback (1983); Rammah \& Hirschmann (1988) e Eisenback
\& Hirschmann-Triantaphyllou (1991). Para análise do fenótipo da isoenzima esterase utilizou-se técnica proposta por Carneiro \& Almeida (2001). Após a identificação, as espécies foram inoculadas em tomateiros 'Rutgers', os quais foram mantidos separadamente em casa-de-vegetação para a produção e manutenção do inóculo. Cerca de 45 dias após a inoculação, ovos e juvenis de segundo estádio (J2) foram extraídos dos sistemas radiculares das plantas para a imediata inoculação do experimento.

Foram realizados dois experimentos, um para a avaliação dos híbridos de porta-enxertos e outro para a avaliação de genótipos elite de pimentas das espécies $C$. chinense, $C$. frutescens e $C$. baccatum. Os genótipos experimentais e testemunhas a serem avaliados foram inicialmente semeados em bandejas de isopor contendo substrato composto de vermiculita expandida, matéria orgânica, macro e micronutrientes. As mudas foram transplantados 30 dias após a semeadura para vasos plásticos com capacidade para $2,0 \mathrm{~L}$, contendo substrato de solo de cerrado, areia lavada, esterco de gado e palha de arroz carbonizada, na proporção de 1:1:1:1 volume a volume. Em seguida, foi realizada a inoculação das plantas com a deposição de 5,0 mL de suspensão em água, contendo 5.000 ovos e J2 de cada espécie, nas raízes ao redor do coleto das plantas. Foram inoculadas isoladamente as seguintes espécies de nematoide-das-galhas: $M$. incognita raça1, M. javanica e $M$. enterolobii.

O primeiro experimento foi conduzido em delineamento inteiramente casualizado em esquema fatorial $2 \times 3$ [dois híbridos: 'Híbrido Experimental (HE)' e Híbrido Experimental Recíproco (HER)'] e três espécies de nematoide-das-galhas (M. incognita raça $1, M$. javanica e $M$. enterolobii), com seis repetições e parcelas compostas por uma planta. Como testemunha suscetível a $M$. incognita raça 1 e resistente a $M$. javanica foi utilizado o híbrido de pimentão 'Magali R', e como testemunha resistente a $M$. incognita raça 1 e $M$. javanica foi utilizado o porta-enxerto 'Silver'.

O segundo experimento foi conduzido em delineamento inteiramente casualizado em esquema fatorial $13 \times 3$, sendo quatro genótipos de $C$. chinense ('CNPH 15.179', 'CNPH 15.185', 'CNPH 15.192' e 'CNPH 15.193'), quatro de C. frutescens ('CNPH 20.148', 'CNPH 20.155', 'CNPH 20.165' e 'CNPH 20.171'), quatro de C. baccatum ('CNPH 046', 'CNPH 0989', 'CNPH 1397' e 'CNPH 2655'), o Híbrido Experimental (HE) e três espécies de nematoide-das-galhas, ( $M$. incognita raça 1, M. javanica e $M$. enterolobii), com seis repetições e parcelas compostas por uma planta. Como testemunha suscetível a $M$. incognita raça 1 e resistente a M. javanica foi utilizado o híbrido de pimentão 'Magali R', e como testemunha resistente a $M$. incognita raça 1 e $M$. javanica foi utilizado o porta-enxerto 'Snooker'. Em ambos os experimentos, para confirmar a viabilidade do inóculo foi utilizado tomateiro cultivar Rutgers.

As variáveis nematológicas, índice de massa de ovos (IMO), índice de galhas (IG), número de ovos por grama de raízes (NOGR) e fator de reprodução (FR) foram avaliadas 70 dias após a inoculação. As variáveis sintomatológicas IMO e IG foram avaliadas para auxiliarem na interpretação dos resultados em relação ao NOGR e FR.

As plantas foram retiradas dos vasos e coletadas separadamente em recipientes identificados. Os sistemas radiculares foram lavados em água corrente e as massas de ovos coloridas conforme metodologia de Dickson \& Struble (1965). Em seguida, procedeu-se a contagem do número de massa de ovos dos nematoides presentes no sistema radicular das plantas, separadamente, em microscópio estereoscópico. O IMO foi obtido conforme Huang et al. (1986), utilizando escala de notas de 1 a 5 (1= raízes sem massa de ovos; $2=$ raízes com 1 a 5 massas de ovos; $3=$ raízes com 6 a 15 massas de ovos; 4= raízes com 16 a 30 massas de ovos e; $5=$ raízes com mais de 30 massas de ovos). Para avaliação do IG, o número de galhas presentes nos sistemas radiculares das plantas foi quantificado separadamente. O IG foi determinado pela escala de 1 a $5(1=$ raiz sem galhas; $2=$ raiz com até 10 galhas pequenas; $3=$ raiz com até 50 galhas pequenas; 4= raiz com mais de 50 galhas pequenas e até 10 galhas grandes; e 5= 
raiz com mais de 50 galhas pequenas e mais de 10 galhas grandes), de acordo com Charchar et al. (2003). Galhas com mais de $3 \mathrm{~mm}$ foram consideradas grandes. Para avaliação do NOGR, as raízes de todos os tratamentos foram lavadas, secas em temperatura ambiente e pesadas antes de serem processadas de acordo com a técnica de Hussey \& Barker (1973), modificada por Boneti \& Ferraz (1981). O FR dos nematoides nos diferentes genótipos foi obtido pela divisão entre as densidades populacionais finais e iniciais $(\mathrm{FR}=\mathrm{Pf} / \mathrm{Pi})$ (Oostenbrink, 1966). Foi considerado como população inicial (Pi) o inóculo extraído, quantificado e calibrado para conter 5.000 ovos e J2 por vaso. Para avaliação da resistência, foram consideradas plantas imunes (I) aquelas com $\mathrm{FR}=0$, plantas resistentes $(\mathrm{R}): \mathrm{FR}<1$; e plantas suscetíveis ( $\mathrm{S})$ : $\mathrm{FR}>1$.

As análises estatísticas dos dados foram realizadas no programa estatístico Genes (v. 2006.4.1) (Cruz, 2006), e as médias agrupadas pelo teste de Scott \& $\operatorname{Knott}(\mathrm{p} \leq 0,05)$.

\section{RESULTADOS E DISCUSSÃO}

Plantas inoculadas com as três espécies de Meloidogyne apresentaram as primeiras galhas nas raízes aos 1520 dias após a inoculação. Já na parte aérea de plantas inoculadas com M. enterolobii foi observado amarelecimento e redução de crescimento das plantas de pimentão aos 40-45 dias após a inoculação, o que não foi observado em plantas inoculadas com $M$. incognita raça 1 e $M$.javanica. Este sintoma pode estar relacionado à maior virulência desta espécie em relação às demais.

A inoculação realizada nos experimentos foi bem sucedida, visto que houve excelente multiplicação das três espécies de nematoides no tomateiro 'Rutgers', o qual apresentou fatores de reprodução $(\mathrm{Pf} / \mathrm{Pi})$ elevados, de 4,54 a 78,00 (primeiro experimento) e 13,62 a 74,46 (segundo experimento) (Tabelas 1 e 2$)$.

Como esperado, o híbrido Magali R comportou-se como suscetível a $M$. incognita raça 1 nos dois experimentos, com fatores de reprodução de 14,29 e

Tabela 1. Reação de híbridos experimentais de Capsicum aos nematoides-das-galhas (Meloidogyne incognita raça 1, M. javanica e M. enterolobii) [reaction of Capsicum experimental hybrids to root-knot nematode (Meloidogyne incognita race 1, M. javanica and M. enterolobii)]. Brasília, Embrapa Hortaliças, 2011.

\begin{tabular}{|c|c|c|c|c|c|}
\hline \multirow{2}{*}{ Genótipos } & \multicolumn{5}{|c|}{ Meloidogyne incognita raça 1} \\
\hline & IMO $^{1}$ & $\mathbf{I G}^{2}$ & NOGR $^{3}$ & $\mathbf{F R}^{4}$ & Reação $^{5}$ \\
\hline $\mathrm{HE}^{9}$ & $2,48 \mathrm{~b}$ & $1,82 \mathrm{c}$ & $144,77 \mathrm{c}$ & $0,65 \mathrm{c}$ & $\mathrm{R}$ \\
\hline $\mathrm{HER}^{10}$ & $1,93 \mathrm{c}$ & $1,00 \mathrm{~d}$ & $254,84 \mathrm{c}$ & $0,72 \mathrm{c}$ & $\mathrm{R}$ \\
\hline Magali $\mathrm{R}^{6}$ & $5,00 \mathrm{a}$ & $2,65 \mathrm{~b}$ & $7.978,85 \mathrm{a}$ & $14,29 \mathrm{~b}$ & $\mathrm{~S}$ \\
\hline Silver ${ }^{7}$ & $2,48 \mathrm{~b}$ & $1,15 \mathrm{~d}$ & $923,77 \mathrm{~b}$ & $0,52 \mathrm{c}$ & $\mathrm{R}$ \\
\hline \multirow[t]{2}{*}{ Rutgers $^{8}$} & $5,00 \mathrm{a}$ & $4,83 \mathrm{a}$ & $4.508,18 \mathrm{a}$ & $19,02 \mathrm{a}$ & $\mathrm{S}$ \\
\hline & \multicolumn{5}{|c|}{ Meloidogyne javanica } \\
\hline $\mathrm{HE}^{9}$ & $1,15 \mathrm{c}$ & $1,00 \mathrm{~b}$ & $24,71 \mathrm{~d}$ & $0,11 \mathrm{c}$ & $\mathrm{R}$ \\
\hline $\mathrm{HER}^{10}$ & $1,00 \mathrm{c}$ & $1,00 \mathrm{~b}$ & $18,17 \mathrm{~d}$ & $0,08 \mathrm{c}$ & $\mathrm{R}$ \\
\hline Magali R ${ }^{6}$ & $1,78 \mathrm{~b}$ & $1,00 \mathrm{~b}$ & $141,30 \mathrm{~b}$ & $0,23 \mathrm{~b}$ & $\mathrm{R}$ \\
\hline Silver ${ }^{7}$ & $1,00 \mathrm{c}$ & $1,00 \mathrm{~b}$ & $38,44 \mathrm{c}$ & $0,08 \mathrm{c}$ & $\mathrm{R}$ \\
\hline \multirow[t]{2}{*}{ Rutgers $^{8}$} & $5,00 \mathrm{a}$ & $5,00 \mathrm{a}$ & $9.249,06 \mathrm{a}$ & $78,00 \mathrm{a}$ & $\mathrm{S}$ \\
\hline & \multicolumn{5}{|c|}{ Meloidogyne enterolobii } \\
\hline $\mathrm{HE}^{9}$ & $5,00 \mathrm{a}$ & $3,82 \mathrm{~d}$ & $3.504,07 \mathrm{~b}$ & $3,80 \mathrm{~b}$ & $\mathrm{~S}$ \\
\hline $\mathrm{HER}^{10}$ & $5,00 \mathrm{a}$ & $4,47 \mathrm{~b}$ & $4.973,28 \mathrm{~b}$ & $5,01 \mathrm{~b}$ & $\mathrm{~S}$ \\
\hline Magali $\mathrm{R}^{6}$ & $5,00 \mathrm{a}$ & $5,00 \mathrm{a}$ & $7.457,08 \mathrm{a}$ & $10,87 \mathrm{a}$ & $\mathrm{S}$ \\
\hline Silver $^{7}$ & $5,00 \mathrm{a}$ & $4,16 \mathrm{c}$ & $9.342,09 \mathrm{a}$ & $3,90 \mathrm{~b}$ & $\mathrm{~S}$ \\
\hline Rutgers $^{8}$ & $3,98 \mathrm{~b}$ & $5,00 \mathrm{a}$ & $829,93 \mathrm{c}$ & $4,54 \mathrm{~b}$ & $\mathrm{~S}$ \\
\hline Média geral & 3,04 & 2,87 & $6.114,16$ & 10,31 & \\
\hline $\mathrm{CV}(\%)$ & 2,05 & 4,52 & 92,09 & 26,45 & \\
\hline
\end{tabular}

${ }^{1} \mathrm{IMO}$ : índice de massa de ovos (eggs mass index); ${ }^{2} \mathrm{IG}$ : índice de galhas (gall index); ${ }^{3} \mathrm{NGOR}$ : número de ovos por grama de raiz (number of eggs per gram of root); ${ }^{4} \mathrm{FR}$ : fator de reprodução (reproduction factor); ${ }^{5}$ Reações de resistência (R: plantas resistentes; S: plantas suscetíveis) [resistance reactions (R: resistant plants; S: susceptible plants)]; ${ }^{6}$ Controle suscetível a $M$. incognita raça 1 (susceptible control to $M$. incognita race $1{ }^{7}$ Controle resistente (resistant control); ${ }^{8}$ Controle padrão de suscetibilidade (susceptible standard control); ${ }^{9} \mathrm{HE}$ (Híbrido Experimental) (Experimental Hybrid); ${ }^{10} \mathrm{HER}$ (Híbrido Experimental Recíproco) (Experimental Reciprocal Hybrid). Dados transformados para log $(\mathrm{x}+1)$ [data transformed to $\log (\mathrm{x}+1)]$. Médias seguidas de mesma letra na coluna não diferem entre si pelo teste de Scott-Knott $(\mathrm{p} \leq 0,05)$ [means followed by the same letter in column do not differ by Scott-Knott $(\mathrm{p} \leq 0.05)]$.

23,05, e resistente a $M$. javanica, com fatores de reprodução de 0,23 e 0,49 (Tabelas 1 e 2). Os porta-enxertos 'Silver' e 'Snooker' apresentaram resistência a estas duas espécies, com fatores de reprodução inferiores a 1,0 e baixos índices de massas de ovos, galhas e ovos por gramas de raiz. A cultivar Magali e os híbridos 'Silver' e 'Snooker' comportaram-se como materiais suscetíveis a $M$. enterolobii, com fatores de reprodução de 10,87, 3,90 e 11,72, respectivamente. Segundo Fargette (1987), M. enterolobii é capaz de se multiplicar em plantas resistentes a outras espécies do gênero Meloidogyne, como o tomate 'Rossol' (gene $\mathrm{Mi}$ ), a soja 'Forest', e a batata-doce 'CDH'.
No primeiro experimento, os híbridos 'Experimental (HE)' e 'Experimental Recíproco (HER)' apresentaram resistência a $M$. incognita raça 1 e $M$. javanica, com fatores de reprodução menores que 1,0 (Tabela 1). Adicionalmente, foram observadas diferenças e semelhanças entre os híbridos experimentais suscetíveis e resistentes utilizados como testemunhas. Os híbridos apresentaram IMO inferiores à da cultivar suscetível Magali R e semelhantes ao porta-enxerto 'Silver'. Um menor IG e NOGR também foi observado nos híbridos inoculados com $M$. incognita raça 1 e $M$. javanica. Com relação a M. enterolobii, verificou-se que estes híbridos comportaram-se como susce- 
Tabela 2. Reação de genótipos de Capsicum aos nematoides-das-galhas (Meloidogyne incognita raça 1, M. javanica e $M$. enterolobii) [reaction of Capsicum genotypes to rootknot nematode (Meloidogyne incognita race 1, M. javanica and M. enterolobii)]. Brasília, Embrapa Hortaliças, 2011.

\begin{tabular}{|c|c|c|c|c|c|c|}
\hline \multirow{2}{*}{ Genótipos } & & \multicolumn{5}{|c|}{ Meloidogyne incognita raça 1} \\
\hline & & IMO $^{1}$ & $I^{2}$ & NOGR $^{3}$ & $\mathbf{F R}^{4}$ & Reação $^{5}$ \\
\hline CNPH 15.179 & \multirow{4}{*}{ C. chinense } & $4,50 \mathrm{a}$ & $4,67 \mathrm{a}$ & $4.203,99 \mathrm{c}$ & $16,03 \mathrm{c}$ & $\mathrm{S}$ \\
\hline CNPH 15.185 & & $4,83 \mathrm{a}$ & $4,67 \mathrm{a}$ & $4.968,85 \mathrm{~b}$ & $19,50 \mathrm{~b}$ & $\mathrm{~S}$ \\
\hline CNPH 15.192 & & $4,83 \mathrm{a}$ & $5,00 \mathrm{a}$ & $2.507,56 \mathrm{c}$ & $12,21 \mathrm{c}$ & $\mathrm{S}$ \\
\hline CNPH 15.193 & & $2,83 \mathrm{~b}$ & $4,00 \mathrm{a}$ & $3.251,91 \mathrm{c}$ & $4,12 \mathrm{~d}$ & $\mathrm{~S}$ \\
\hline CNPH 20.148 & \multirow{4}{*}{ C. frutescens } & $1,67 \mathrm{c}$ & $1,50 \mathrm{c}$ & $214,50 \mathrm{~d}$ & $0,78 \mathrm{e}$ & $\mathrm{R}$ \\
\hline CNPH 20.155 & & $1,67 \mathrm{c}$ & $2,17 \mathrm{~b}$ & $68,89 \mathrm{~d}$ & $0,35 \mathrm{e}$ & $\mathrm{R}$ \\
\hline CNPH 20.165 & & $2,17 \mathrm{~b}$ & $1,83 \mathrm{~b}$ & $184,43 \mathrm{~d}$ & $0,60 \mathrm{e}$ & $\mathrm{R}$ \\
\hline CNPH 20.171 & & $1,33 \mathrm{~d}$ & $1,33 \mathrm{c}$ & $91,57 \mathrm{~d}$ & $0,26 \mathrm{e}$ & $\mathrm{R}$ \\
\hline CNPH 046 & \multirow{4}{*}{ C. baccatum } & $4,50 \mathrm{a}$ & $4,83 \mathrm{a}$ & $2.994,71 \mathrm{c}$ & $16,87 \mathrm{c}$ & $\mathrm{S}$ \\
\hline CNPH 0989 & & $4,83 \mathrm{a}$ & $5,00 \mathrm{a}$ & $4.477,97 \mathrm{~b}$ & $13,35 \mathrm{c}$ & $\mathrm{S}$ \\
\hline CNPH 1397 & & $5,00 \mathrm{a}$ & $5,00 \mathrm{a}$ & $2.837,04 \mathrm{c}$ & $12,25 \mathrm{c}$ & S \\
\hline CNPH 2655 & & $4,50 \mathrm{a}$ & $4,50 \mathrm{a}$ & $5.141,15 \mathrm{~b}$ & $24,27 \mathrm{~b}$ & $\mathrm{~S}$ \\
\hline $\mathrm{HE}^{9}$ & & $1,83 \mathrm{c}$ & $1,33 \mathrm{c}$ & $152,44 \mathrm{~d}$ & $0,61 \mathrm{e}$ & $\overline{\mathrm{R}}$ \\
\hline Magali $\mathrm{R}^{6}$ & & $4,50 \mathrm{a}$ & $4,83 \mathrm{a}$ & $5.493,47 \mathrm{~b}$ & $23,05 \mathrm{~b}$ & $\mathrm{~S}$ \\
\hline Snooker ${ }^{7}$ & & $1,00 \mathrm{~d}$ & $1,00 \mathrm{c}$ & $310,42 \mathrm{~d}$ & $0,67 \mathrm{e}$ & $\mathrm{R}$ \\
\hline Rutgers $^{8}$ & & $5,00 \mathrm{a}$ & $5,00 \mathrm{a}$ & $7.381,90 \mathrm{a}$ & $38,58 \mathrm{a}$ & $\mathrm{S}$ \\
\hline
\end{tabular}

\begin{tabular}{|c|c|c|c|c|c|c|}
\hline & & \multicolumn{5}{|c|}{ Meloidogyne javanica } \\
\hline CNPH 15.179 & \multirow{4}{*}{ C. chinense } & $3,00 \mathrm{~b}$ & $2,67 \mathrm{~b}$ & $4.243,98 \mathrm{c}$ & $13,70 \mathrm{~d}$ & $\mathrm{~S}$ \\
\hline CNPH 15.185 & & $3,67 \mathrm{~b}$ & $3,50 \mathrm{~b}$ & $5.794,77 \mathrm{c}$ & $21,92 \mathrm{c}$ & $\mathrm{S}$ \\
\hline CNPH 15.192 & & $2,83 \mathrm{~b}$ & $3,00 \mathrm{~b}$ & $4.063,29 \mathrm{c}$ & $14,88 \mathrm{~d}$ & $\mathrm{~S}$ \\
\hline CNPH 15.193 & & $4,17 \mathrm{a}$ & $3,50 \mathrm{~b}$ & $10.203,99 \mathrm{a}$ & $16,17 \mathrm{~d}$ & $\mathrm{~S}$ \\
\hline CNPH 20.148 & \multirow{4}{*}{ C. frutescens } & $1,17 \mathrm{c}$ & $1,50 \mathrm{c}$ & $291,04 \mathrm{e}$ & $0,58 \mathrm{e}$ & $\bar{R}$ \\
\hline CNPH 20.155 & & $1,00 \mathrm{c}$ & $1,17 \mathrm{c}$ & $336,88 \mathrm{e}$ & $0,39 \mathrm{e}$ & $\mathrm{R}$ \\
\hline CNPH 20.165 & & $1,17 \mathrm{c}$ & $1,33 \mathrm{c}$ & $194,69 \mathrm{e}$ & $0,54 \mathrm{e}$ & $\mathrm{R}$ \\
\hline CNPH 20.171 & & $1,67 \mathrm{c}$ & $1,83 \mathrm{c}$ & $1.832,27 \mathrm{~d}$ & $2,26 \mathrm{e}$ & $\mathrm{S}$ \\
\hline CNPH 046 & \multirow{4}{*}{ C. baccatum } & $4,83 \mathrm{a}$ & $4,67 \mathrm{a}$ & $8.561,65 \mathrm{~b}$ & $56,37 \mathrm{~b}$ & $\bar{S}$ \\
\hline CNPH 0989 & & $3,67 \mathrm{a}$ & $3,50 \mathrm{a}$ & $4.621,49 \mathrm{~b}$ & $14,10 \mathrm{c}$ & $\mathrm{S}$ \\
\hline CNPH 1397 & & $3,67 \mathrm{~b}$ & $3,50 \mathrm{~b}$ & $4.621,49 \mathrm{c}$ & $14,10 \mathrm{~d}$ & $\mathrm{~S}$ \\
\hline CNPH 2655 & & $3,00 \mathrm{~b}$ & $3,00 \mathrm{~b}$ & $5.245,39 \mathrm{c}$ & $29,97 \mathrm{c}$ & $\mathrm{S}$ \\
\hline $\mathrm{HE}^{9}$ & & $0,67 \mathrm{~d}$ & $0,67 \mathrm{~d}$ & $0,00 \mathrm{a}$ & $0,00 \mathrm{e}$ & $\bar{I}$ \\
\hline Magali $\mathrm{R}^{6}$ & & $0,83 \mathrm{c}$ & $0,83 \mathrm{~d}$ & $207,23 \mathrm{e}$ & 0,49 e & $\mathrm{R}$ \\
\hline Snooker $^{7}$ & & $0,17 \mathrm{e}$ & $0,33 \mathrm{~d}$ & $137,65 \mathrm{e}$ & $0,06 \mathrm{e}$ & $\mathrm{R}$ \\
\hline Rutgers $^{8}$ & & $5,00 \mathrm{a}$ & $5,00 \mathrm{a}$ & $10.792,11 \mathrm{a}$ & $74,46 \mathrm{a}$ & $\mathrm{S}$ \\
\hline
\end{tabular}

\begin{tabular}{|c|c|c|c|c|c|c|}
\hline & & \multicolumn{5}{|c|}{ Meloidogyne enterolobii } \\
\hline CNPH 15.179 & & $3,50 \mathrm{~b}$ & $4,50 \mathrm{~b}$ & $2.788,30 \mathrm{c}$ & $6,43 \mathrm{c}$ & $\mathrm{S}$ \\
\hline CNPH 15.185 & & $4,50 \mathrm{a}$ & $4,83 \mathrm{a}$ & $4.220,95 \mathrm{~b}$ & $16,18 b$ & $\mathrm{~S}$ \\
\hline CNPH 15.192 & c. chinense & $4,17 \mathrm{~b}$ & $4,33 \mathrm{~b}$ & $1.919,73 \mathrm{c}$ & $8,04 \mathrm{c}$ & $\mathrm{S}$ \\
\hline CNPH 15.193 & & $4,00 \mathrm{~b}$ & $5,00 \mathrm{a}$ & $6.516,64 \mathrm{a}$ & $7,50 \mathrm{c}$ & $\mathrm{S}$ \\
\hline CNPH 20.148 & & $3,50 \mathrm{~b}$ & $5,00 \mathrm{a}$ & $1.071,68 \mathrm{c}$ & $2,41 \mathrm{~d}$ & $\mathrm{~S}$ \\
\hline CNPH 20.155 & & $4,33 \mathrm{a}$ & $5,00 \mathrm{a}$ & $1.103,53 \mathrm{c}$ & $3,32 \mathrm{~d}$ & $\mathrm{~S}$ \\
\hline CNPH 20.165 & C. frutescens & $3,83 \mathrm{~b}$ & $4,17 \mathrm{~b}$ & $2.201,49 \mathrm{c}$ & $2,22 \mathrm{~d}$ & $\mathrm{~S}$ \\
\hline CNPH 20.171 & & $3,50 \mathrm{~b}$ & $5,00 \mathrm{a}$ & $1.772,95 \mathrm{c}$ & $4,13 \mathrm{~d}$ & $\mathrm{~S}$ \\
\hline CNPH 046 & & $\overline{5,00 a}$ & $5,00 \mathrm{a}$ & $5.268,39 \mathrm{~b}$ & $28,42 \mathrm{a}$ & $\bar{S}$ \\
\hline CNPH 0989 & & $5,00 \mathrm{a}$ & $5,00 \mathrm{a}$ & $8.450,20 \mathrm{a}$ & $19,80 \mathrm{~b}$ & $\mathrm{~S}$ \\
\hline CNPH 1397 & C. baccatum & $5,00 \mathrm{a}$ & $5,00 \mathrm{a}$ & $7.301,17 \mathrm{a}$ & $35,03 \mathrm{a}$ & $\mathrm{S}$ \\
\hline CNPH 2655 & & $4,83 \mathrm{a}$ & $4,83 \mathrm{a}$ & $5.442,71 \mathrm{~b}$ & $21,98 \mathrm{~b}$ & $\mathrm{~S}$ \\
\hline $\mathrm{HE}^{9}$ & & $4,83 \mathrm{a}$ & $5,00 \mathrm{a}$ & $5.157,35 \mathrm{~b}$ & $16,95 \mathrm{~b}$ & $\mathrm{~S}$ \\
\hline Magali $\mathrm{R}^{6}$ & & $5,00 \mathrm{a}$ & $5,00 \mathrm{a}$ & $7.281,46 \mathrm{a}$ & $18,88 \mathrm{~b}$ & $\mathrm{~S}$ \\
\hline Snooker $^{7}$ & & $4,83 \mathrm{a}$ & $5,00 \mathrm{a}$ & $5.983,48 \mathrm{~b}$ & $11,72 \mathrm{c}$ & $\mathrm{S}$ \\
\hline Rutgers $^{8}$ & & $3,67 \mathrm{~b}$ & $5,00 \mathrm{a}$ & $4.057,59 \mathrm{~b}$ & $13,62 \mathrm{~b}$ & $\mathrm{~S}$ \\
\hline Média geral & & 3,44 & 3,64 & $3.1722,35$ & 13,99 & \\
\hline CV (\%) & & 7,51 & 6,22 & 23,02 & 21,01 & \\
\hline
\end{tabular}

${ }^{1} \mathrm{IMO}$ : índice de massa de ovos (eggs mass index); ${ }^{2} \mathrm{IG}$ : índice de galhas (gall index); ${ }^{3} \mathrm{NGOR}$ : número de ovos por grama de raiz (number of eggs per gram of root); ${ }^{4} \mathrm{FR}$ : fator de reprodução (reproduction factor); ${ }^{5}$ Reações de resistência (I: plantas imunes, R: plantas resistentes e S: plantas suscetíveis) [resistance reactions (I: immune plants, R: resistant plants and S: susceptible plants)]; ${ }^{6}$ Controle suscetível a $M$. incognita raça 1 (susceptible control to $M$. incognita race 1 ); ${ }^{7}$ Controle resistente (resistant control); ${ }^{8}$ Controle padrão de suscetibilidade (susceptible standard control) $\mathrm{HE}^{9}=$ 'híbrido experimental' (experimental hybrid)'. Dados transformados para $\log (\mathrm{x}+1)$ [data transformed to $\log (\mathrm{x}+1)]$. Médias seguidas de mesma letra na coluna não diferem entre si pelo teste de Scott-Knott $(p \leq 0,05)$ [means followed by the same letter in column do not differ by Scott-Knott $(\mathrm{p} \leq 0.05)]$. tíveis, com fatores de reprodução de 3,80 e 5,01, respectivamente. Estes apresentaram IMO semelhantes aos da cultivar Magali R e do porta-enxerto hibrido 'Silver'.

A diferença na suscetibilidade dos híbridos avaliados em relação às espécies de Meloidogyne possivelmente se deve a especificidade de alguns genes $M e$ de resistência em pimentão a espécies de Meloidogyne (Djian-Caporalino et al., 1999). A resistência a $M$. enterolobii aparentemente é mediada por genes diferentes dos que conferem resistência a outras espécies e raças de Meloidogyne (Melo et al., 2011). Todavia, considerando todos os parâmetros utilizados, os híbridos experimentais tiveram comportamento similar ao porta-enxerto 'Silver' do ponto de vista de resistência a nematoides. Entretanto, o hibrido experimental leva vantagem em relação ao 'Silver' por apresentar resistência à murcha bacteriana (Ralstonia solanacearum) (Sakata, 2012).

Resultados semelhantes foram observados por outros autores após a avaliação de genótipos de pimentão quanto à resistência a Meloidogyne spp. Santos et al. (2002) avaliaram porta-enxertos híbridos de pimentão quanto a resistência a $M$. incognita raça 2 e observaram que os híbridos 'AF 2638' e 'AF 2640' comportaram-se como resistentes quando comparados com o padrão de suscetibilidade utilizado, no caso o híbrido 'Elisa'. Segundo os autores, os porta-enxertos 'AF 2638' e 'AF 2640' apresentaram baixos fatores de reprodução, 0,007 e 0,003 respectivamente, podendo estes ser utilizados em áreas infestadas com $M$. incognita raça 2. Peixoto et al. (1999) avaliaram diferentes genótipos de pimentão quanto a resistência a $M$. incognita raça 2 e $M$. javanica e verificaram que todas as linhagens experimentais e cultivares avaliadas foram resistentes a $M$. javanica e suscetíveis a $M$. incognita raça 2. O mesmo foi observado para os híbridos F1 testados; porém, o grau de resistência apresentado a $M$. javanica foi menor que nas linhagens.

Vale ressaltar que os híbridos experimentais avaliados neste trabalho se destacaram no Programa de Melhoramento de Capsicum da Embrapa por 
apresentarem resistência a outros patógenos de solo importantes em pimentão, como Ralstonia solanacearum e Phytophthora capsici. Mesmo apresentando suscetibilidade a $M$. enterolobii, estes poderão ser eficientemente utilizados como porta-enxertos em áreas de cultivo protegido infestadas com $M$. incognita raça 1 e $M$. javanica, que são espécies prevalecentes em áreas de produção de hortaliças nas principais regiões do pais.

No segundo experimento verificou-se que todos os genótipos de $C$. chinense e $C$. baccatum foram suscetíveis às três espécies de Meloidogyne avaliadas (Tabela 2). Os genótipos de C. frutescens apresentaram resistência a $M$. incognita raça 1 e $M$. javanica e suscetibilidade a $M$. enterolobii, exceto 'CNPH 20.171' que também apresentou suscetibilidade a $M$. javanica. Genótipos de $C$. frutescens inoculados com $M$. incognita raça 1 e $M$. javanica apresentaram IMO, IG e NOGR inferiores aos genótipos de $C$. chinense e $C$. baccatum e a cultivar Magali R. Já genótipos de $C$. frutescens inoculados com $M$. javanica apresentaram IMO, IG e NOGR inferiores aos genótipos de $C$. chinense e $C$. baccatum e semelhantes a cultivar Magali R. Resultados semelhantes foram observados por Oliveira et al. (2009) ao avaliaram três porta-enxertos de C. annuum, um porta-enxerto de C. frutescens e nove de C. chinense quanto a resistência a $M$. incognita. Os autores verificaram que somente os genótipos de C. annuum e C. frutescens foram resistentes a $M$. incognita. Melo et al. (2011) avaliaram acessos de $C$. chinense (BGH-433 e BGH-4285) e verificaram que estes apresentaram índices de reprodução abaixo de $25 \%$ e inferiores aos observados em genótipos de $C$. annuum. Segundo os autores, essa resistência moderada a $M$. enterolobii não parece estar associada à resistência a M. incognita, pois BGH-433 (resistente a $M$. incognita) e BGH-4285 (suscetível) apresentaram graus semelhantes de resistência a $M$. enterolobii. Resultados similares foram obtidos por Oliveira (2007) que, ao avaliar diferentes espécies do gênero Capsicum, observou que todos os nove genótipos de $C$. chinense foram suscetíveis a $M$. enterolobii, com fatores de reprodução de 2,74 a 12,62, menores que os obtidos em C. annuum, no mesmo experimento, no qual somente o genótipo de $C$. frutescens foi considerado resistente.

No Brasil, até o presente, não existem genótipos de Capsicum resistentes a $M$. enterolobii, o que torna necessário a busca por porta-enxertos resistentes a esta espécie, bem como o desenvolvimento e a adoção de estratégias de controle para evitar a infestação de novas áreas. Em hortaliças, relatos de danos causados por $M$. enterolobii têm sido frequentes.

\section{REFERÊNCIAS}

BONETI JIS; FERRAZ S. 1981. Modificações do método de Hussey \& Barker para extração de ovos de Meloidogyne exigua em raízes de cafeeiro. Fitopatologia Brasileira 6: 553.

CARNEIRO RMDG; ALMEIDA MRA. 2001. Técnica de eletroforese usada no estudo de enzimas dos nematoides de galhas para identificação de espécies. Nematologia Brasileira 25: 35-44.

CARNEIRO RMDG; ALMEIDA MRA; BRAGA RS; ALMEIDA CA; GIORIA R. 2006. Primeiro registro de Meloidogyne mayaguensis parasitando plantas de tomate e pimentão resistentes à meloidoginose no estado de São Paulo. Nematologia Brasileira 30: 81-86.

CARNEIRO RMDG; MOREIRA WA; ALMEIDA MRA; GOMES ACMM. 2001. Primeiro registro de Meloidogyne mayaguensis em goiabeira no Brasil. Nematologia Brasileira 25: 223-232.

CHARCHAR JM; GONZAGA V; GIORDANO LB; BOITEUX LS; REIS NVB; ARAGÃO FAS. 2003. Reações de cultivares de tomate à infecção por população mista de Meloidogyne incognita raça 1 e $M$. javanica em estufa plástica e campo. Nematologia Brasileira 27: 49-54.

CRUZ CD. 2006. Programa Genes: estatística experimental e matrizes. Viçosa: UFV. 285p.

DICKSON DW; STRUBLE FB. 1965. A sievingstaining technique for extraction of egg mass of Meloidogyne incognita from soil. Phytopathology 55: 497.

DJIAN-CAPORALINO C; PIJAROWSKI L; JANUEL A; LEFEBVRE V; DAUBEZA A; PALLOOIX A; DALMASSO A; ABAD P. 1999. Spectrum of resistance to root-knot nematodes and inheritance of heat-stable resistance in pepper (Capsicum annuиm). Theoretical and Applied Genetics 99: 496-502.

EISENBACK JD; H HIRSCHMANNTRIANTAPHYLLOU. 1991. Root-knot nematodes: Meloidogyne species and races. In: WR NICKLE (ed). Manual of Agricultural Nematology New York. 191-274.

FARGETTE M. 1987. Use of esterase phenotype in the taxonomy of the genus Meloidogyne. 2. Esterase phenotypes observed in West African populations and their characterization. Revue de Nématologie 10: 45-56.

HUANG SP; MIRANDA JEC; MALUF WR. 1986. Resistance to root-knot nematodes in a Brazilian sweet potato collection. Fitopatologia Brasileira 11: 761-767.

HUSSEY RS; BARKER KR. 1973. A comparasion of methods of collecting inocula of Meloidogyne spp. including a new technique. Plant Disease Report 57: 1025-1028.

MELO OD; MALUF WR; GONÇALVES RJS; NETO ACG; GOMES LAA; CARVALHO RC. 2011. Triagem de genótipos de hortaliças para resistência a Meloidogyne enterolobii. Pesquisa Agropecuária Brasileira. 46: 829835.

OLIVEIRA CD. 2007. Enxertia de plantas de pimentão em Capsicum spp. no manejo de nematóides de galha. Jaboticabal: UNESP. $134 \mathrm{p}$ (Tese doutorado).

OLIVEIRA CD; BRAZ LT; SANTOS JM; BANZATTO DA; OLIVEIRA PR. 2009. Resistência de pimentas a nematóides de galha e compatibilidade enxerto/porta-enxerto entre híbridos de pimentão e pimentas. Horticultura Brasileira 27: 520-526.

OOSTENBRINK M. 1966. Major characteristics of the relation between nematodes and plants. Mededelingen Landbouw 66: 1-46.

PEIXOTO JR; MALUF WR; CAMPO VP. 1999. Avaliação de linhagens, híbridos F1 e cultivares de pimentão quanto à resistência a Meloidogyne spp. Pesquisa Agropecuária Brasileira 34: 2259-2265.

RAMMAH A; HIRSCHMANN H. 1988. Meloidogyne mayaguensis $\mathrm{n}$. $\mathrm{sp}$. (Meloidogynidae), a root-knot nematode from Puerto Rico. Journal of Nematology 20: 58-69.

SAKATA - Seeds Sudameria Ltda. Silver: pimentão - porta enxerto. 2012, 03 de abril. Disponível em: http://www.sakata.com.br/ index.php?action $=$ catalogo \&cultura $=4 \&$ prod uto $=1098 \&$ language $=$ pt.

SANTOS HS. 2003. Histórico da enxertia em Hortaliças: utilização e pesquisa. In: GOTO R; SANTOS HS; GAÑIZARES KAL (eds). Enxertia em hortaliças. São Paulo: UNESP, cap. 2. p.11-14.

SANTOS HS; WILCKEN SRS; GOTO R. 2002. Reprodução de Meloidogyne incognita raça 2 em diferentes porta-enxertos de pimentão (Capsicum annuum) Nematologia Brasileira 26: 209-211.

SYNGENTA. Pimentão Snooker - Porta Enxerto. 2012, 02 de abril. Disponível em: http://www. syngenta.com/country/br/pt/produtosemarcas/ sementes/vegetais/Pages/pimentao-snookerporta-enxerto.aspx.

YANG B; EISENBACK JD. 1983. Meloidogyne enterolobii sp. (Meloidogynidae), a root-knot nematode parasitising pacara earpod tree in China. Journal of Nematology 15: 381-391. 